\title{
Photoacoustic characterization of phase transitions in amorphous metal alloys
}

\author{
D.J. Orzi ${ }^{1}$, G.M. Bilmes ${ }^{1}$, J.O. Tocho ${ }^{1}$, N. Mingolo ${ }^{2}$, O.E. Martinez ${ }^{3}$ \\ ${ }^{1}$ Centro de Investigaciones opticas (CIC-CONICET) and Universidad Nacional de La Plata, C.C.124, 1900, La Plata, Argentina \\ Fax: +54-21/71-2771, E-mail: postmaster @ciop.edu.ar) \\ ${ }^{2}$ Departamento de Física, Facultad de Ingeniería, UBA, Paseo Colón 850, 1063 Buenos Aires, Argentina \\ (Fax: +54-1-331-0129, E-mail: nmingol@aleph.fi.uba.ar) \\ ${ }^{3}$ Departamento de Física, FCEyN-UBA, Pabellón 1, Ciudad Universitaria, 1428 Buenos Aires, Argentina \\ (Fax: +54-1-782-7647, E-mail: oem@df.uba.ar)
}

Received: 24 February 1997

\begin{abstract}
In this work a pulsed laser photoacoustic technique is employed in amorphous alloys for the characterization of crystallization temperatures and the determination of the activation energies for the transitions. It is shown that the changes produced in the photoacoustic signal generated by low energy laser pulses $(<200 \mu \mathrm{J})$, is a sensitive probe for detecting = the crystallization of a metallic amorphous sample. A piezoelectric transducer attached to the sample by means of a glass substrate was used for the acoustic detection. The developed technique has the advantages of requiring a minimum amount of sample, with no special sample preparation or conditioning. In order to demonstrate the validity of the technique, it was applied to the study of the crystallization processes of the amorphous $\mathrm{Mg}-\mathrm{Zn}$ alloy, comparing the result with respect to resistivity measurements.
\end{abstract}

PACS: $78.20 ; 61.40 ; 64$

The stability of amorphous metals can be determined by thermal measurements. When an amorphous metal is heated above its glass transition temperature, it undergoes first a second order glass to undercooled liquid transition and afterwards a first order liquid to crystal transition $[1,2]$. The structural changes are usually detected by X-ray or neutron diffraction, scanning calorimetry or electrical resistivity" measurements. The stability of the amorphous phase and the activation energies for the transitions are determined by the kinetics of transformation, that is, the delay before the transition takes place, after heating above the critical temperature. When isothermal annealing is used, the activation energy is determined from Arrhenius plots and at constant heating rates from Kissinger plots [3]. In this work a new technique for the determination of phase transition in metal alloys, based on the photoacoustic effect is presented. Photoacoustics is related to the phenomena of the generation of acoustic waves in a medium after interaction with modulated or pulsed electromagnetic radiation. One interesting property of-the phenomena, is that the photoacoustic signal generated, depends on the thermoelastic parameters of the medium (thermal expansion, sound velocity, heat capacity, etc.), magnitudes that change greatly in a phase transition, hence, structural changes can be easily determined in realtime by monitoring the changes in the photoacoustic signals. Prior work related to photoacoustic characterization of phase transitions relies on the use of a chopped lamp or a CW low power laser and a gas cell with a microphone [4]. This technique requires a very small volume of gas contacting the sample and the microphone [5]. This requirement is difficult to achieve as the microphone must be placed far from the sample, outside the temperature controlled region, leading to sophisticated designs $[6,7]$.

In this work a pulsed laser photoacoustic technique is employed. Pulsed laser generation of acoustic waves in the condensed phase, has been widely used for the characterization of materials [8]. Here, the laser excitation of the sample acts only as a source of acoustic waves, then the energy of the pulse must be always sufficiently low to avoid heating or surface changes. As the detector used is a piezoelectric transducer contacting the sample by means of a glass substrate, it can be easily placed outside the oven without any special requirement in the design.

In order to demonstrate the validity of the technique developed, it was applied to the study of the crystallization processes of the amorphous $\mathrm{Mg}-\mathrm{Zn}$ alloy $\left(\mathrm{Mg}_{70} \mathrm{Zn}_{30}\right)$. This alloy was selected because it has been thoroughly studied and the large number of prior publications allows a reliable comparison with the results. It presents a eutectic at the composition used, and has a high temperature equilibrium phase $\mathrm{Mg}_{51} \mathrm{Zn}_{20}$ (denoted as $\mathrm{Mg}_{7} \mathrm{Zn}_{3}$ ) that has been characterized by Higashi [9]. The sample becomes amorphous after rapid cooling from the melt $[10,11]$ with a short range order similar to that of the $\mathrm{Mg}_{51} \mathrm{Zn}_{20}$ crystalline phase [12-14]. According to Andonov [15] it is actually described by the coexistence of a disordered phase similar to the liquid state and better organized aggregates which are not yet crystallized. The amorphous phase is only marginally "stable" [16] and different authors have found somewhat different transition temperatures, depending on the thermal history of the material $[7,17]$. The crystallization upon heating has been described 
by several authors $[10,11,13,18,19]$, and the activation energy for crystallization has been determined $[11,18]$.

In characterizing this new technique, electrical conductivity and photoacoustic signals were simultaneously measured during different thermal cycles of the samples in the range between room temperature and $560 \mathrm{~K}$, identifying completely the most important phase transitions that take place in this material. Activation energies of the transition as well as the sound velocity in the amorphous and crystalline phase were also measured.

Generation of photoacoustic signals and changes as a function of temperature, are discussed in terms of the changes in the thermodynamic parameters of the samples.

\section{Experimental}

The experimental setup used is shown in Fig. 1. The excitation source was the second harmonic $(\lambda=532 \mathrm{~nm})$ of a Q-switched $\mathrm{Nd}^{3+}$-YAG laser, providing $7 \mathrm{~ns}$ width pulses at a repetition rate of $10 \mathrm{~Hz}$ and attenuated to less than $200 \mu \mathrm{J}$ to avoid excessive heating of the material. The samples were ribbons $20 \mu \mathrm{m}$ thick, $1 \mathrm{~mm}$ wide, and pieces around $1 \mathrm{~mm}$ long were cut and pressed between an L-shaped glass substrate and a glass cover, to ensure good acoustical contact ("sample holder"). The short arm of the substrate was located inside a temperature controlled oven, and the end of the large part outside it, attached with glue to the acoustic transducer. The latter was a cylindrical ceramic piezoelectric detector (PZT) $4 \mathrm{~mm}$ in diameter and $4 \mathrm{~mm}$ long. The substrate was L-shaped in order to avoid spurious acoustic signals produced by scattered light absorbed at the surface of the transducer [20]. The peak to peak amplitude of the first detected acoustic oscillation $(H)$ was monitored as a function of the temperature. The acoustic signals were amplified $(\times 800)$, and afterwards processed by a storage digital oscilloscope that sampled at 100 Msamples/sec with 8-bit resolution per point.

The temperature of the oven could be controlled between room temperature and $560 \mathrm{~K}$ using heating rates between $1 \mathrm{~K} / \mathrm{min}$ and $15 \mathrm{~K} / \mathrm{min}$. The temperature of the sample was measured by a thermocouple, mounted on the glass substrate at a distance of a few millimetres from the sample. Energy measurements $(E)$ were performed with a pyroelectric detector, sampling the pulse using a glass plate as the beam splitter. The four probe method was used for the measurement of the resistivity $(R)$, using samples $10 \mathrm{~mm}$ long and electric contacts made by pressing copper foils.

Automatic and simultaneous measurements of the amplitude of the acoustic signal, energy of the pulse, temperature, and resistivity, were made by taking 32 averages every 10 seconds. The data were stored in the computer for further processing.

The samples ( $\mathrm{Mg}_{70} \mathrm{Zn}_{30}$ near eutectic composition), were manufactured by the melt spinning technique [21], and X-ray diffraction spectra were taken to verify that the material was initially amorphous.

Figure 2 shows the changes observed in the acoustic signal at three different temperatures $(290 \mathrm{~K}, 423 \mathrm{~K}$ and $523 \mathrm{~K})$. The registered signal is the result of the response of the detector to all the waves, including reflections, and presents a characteristic "ringing" of the piezoelectric crystal. Hence, the selection of the part of the signal used to measure, must

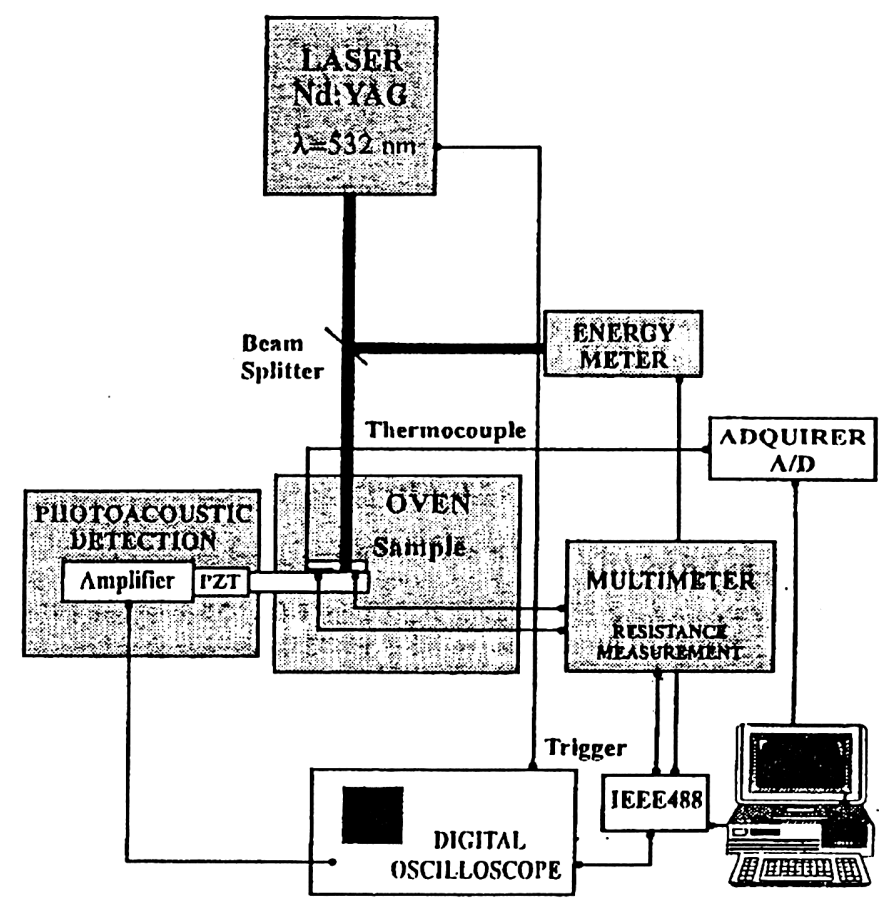

Fig. 1. Experimental set-up used for the photoacoustic detection. Automatic and simultaneous measurements of the amplitude of the acoustic signal, pulse energy, temperature, and resistivity, were made by taking 32 averages every 10 seconds. The data was stored in the computer for further processing

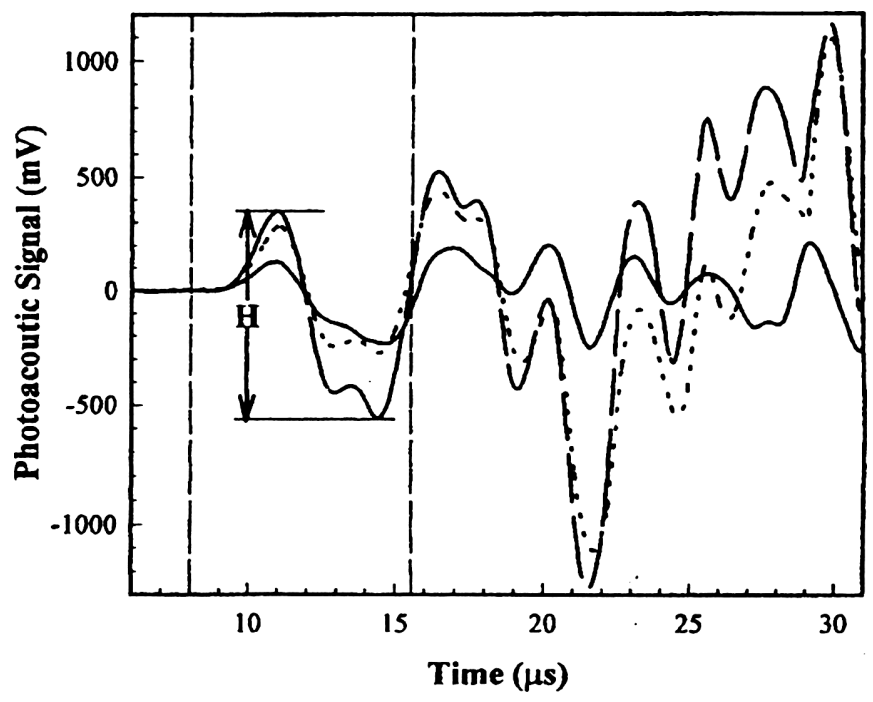

Fig. 2. Time dependence of the photoacoustic signal at different temperatures. (solid) $290 \mathrm{~K}$, (short dash) $423 \mathrm{~K}$; (long dash) $523 \mathrm{~K}$. The change in the shape is evident, and the peak to peak amplitude of the first oscillation is taken as the photoacoustic signal $H$

be made carefully. It is clear that, with the exception of the first part of the signal, the rest not only changes in amplitude but also in shape, probably due to interference effects between different acoustic waves, deformations of the sample, etc. Taking this into account, the peak to peak amplitude of the first oscillation was used as the photoacoustic signal $H$, and was divided by the laser pulse energy $(E)$ to compensate the shot to shot laser fluctuations. 


\section{Results}

Figure 3 shows a simultaneous measurement of the normalized photoacoustic signal $H / E$ and the resistance $R$ as a function of the temperature, performed by heating the samples at a rate of $9 \mathrm{~K} / \mathrm{min}$. In both cases strong changes were observed above $360 \mathrm{~K}$ (an increase of $H / E$; decrease of $R$ ), corresponding to the crystallization of the sample, as it was previously reported in the literature $[10,11,13,18]$. After heating to $570 \mathrm{~K}$, the sample was cooled to room temperature, showing the irreversibility of the transition. Successive temperature cycles of heating and cooling showed no further changes, confirming complete crystallization. Thermal cycles performed from room temperature to $410 \mathrm{~K}$ (after the first transition), showed a similar behaviour consistent with a complete crystallization also at this stage. Hence two transition temperatures can be easily identified, one around $370 \mathrm{~K}$ and the other around $470 \mathrm{~K}$.

To determine the reproducibility of the measurements, experiments were performed with the same heating rate with different pieces of the same ribbon. Similar curves were obtained with a reproducibility within $5 \%$ of the values of the transition temperatures

The activation energies for the crystallization processes were estimated by the Kissinger "peak shift" method [3,11]. This procedure involves plotting $\log \left(T_{i}^{2} / v\right)$ as a function of $1 / T_{i}$, where $T_{\mathrm{i}}$ is the temperature of the transition, and $v$ is the heating rate. The slope of the straight line obtained gives the activation energy of the transition.

Two activation energies were estimated, corresponding to the two steps of the crystallization. Figure 4 shows Kissinger plots corresponding to the data obtained for different heating rates between $1 \mathrm{~K} / \mathrm{min}$ and $15 \mathrm{~K} / \mathrm{min}$. The activation energies obtained for the two transitions are $E_{1}=153 \mathrm{~kJ} / \mathrm{mole}$, and $E_{2}=92 \mathrm{~kJ} / \mathrm{mole}$.

One of the possible sources of variation in the photoacoustic signal is a change in the sound velocity, a parameter that has not been measured previously for this alloy. With the aim of establishing the importance of the different parameters in this change, the sound velocity was determined before and after the crystallization of the sample. This was performed outside the oven by measuring the arrival time of the acoustic signal after the laser shot, as a function of the distance between the laser spot and the transducer. Figure 5 shows that the sound velocity in the amorphous phase $(4767 \pm 50 \mathrm{~m} / \mathrm{s})$ is $15 \%$ smaller than in the crystalline phase $(5562 \pm 77 \mathrm{~m} / \mathrm{s})$.

\section{Discussions}

The origin of the acoustic signal is the pressure wave generated by the local heating of the material, after absorbing part of the laser energy. The signal detected depends in a complicated manner on the excitation-detection geometry conditions of the experiment, and both the surface and bulk waves are coupled to the detector. From the treatment performed by Bunkin et al. for the thermo-optical generation of acoustic waves in a solid [22], the following general expression for the amplitude of the acoustic pulse as a function of the thermo-

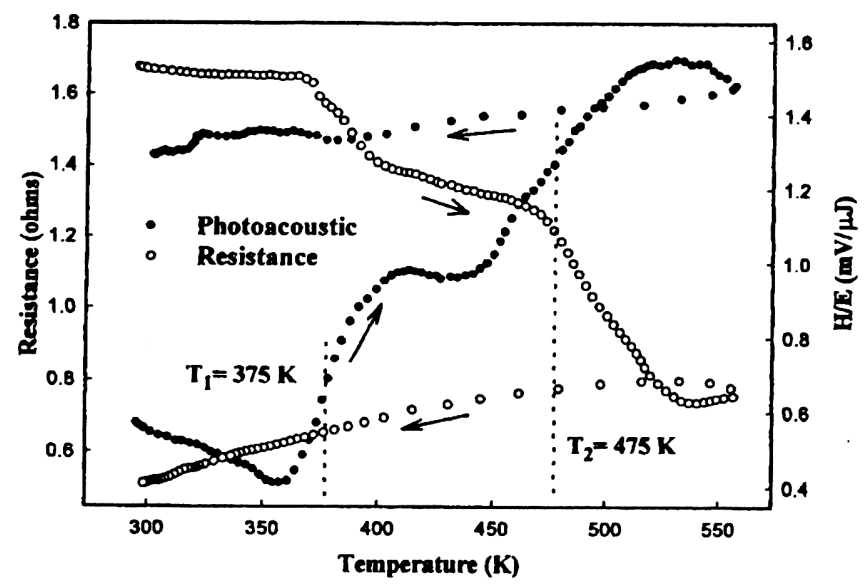

Fig. 3. Simultaneous measurement of resistance $(R)$ and normalized photoacoustic signal $(H / E)$, as a function of temperature for a $\mathrm{Mg}_{70} \mathrm{Zn}_{30}$ alloy. Arrows show the heating $(\rightarrow)$ and cooling $(\leftrightarrow$ cycles respectively. The heating rate was $9 \mathrm{~K} / \mathrm{min}$. Two transitions appear. The transition temperature was taken at the half point of the signal change, as shown

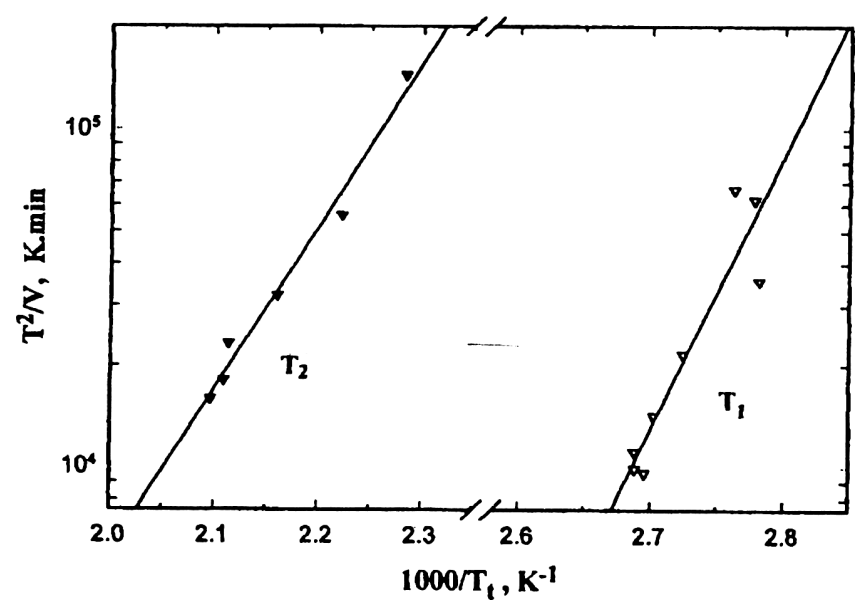

Fig. 4. Kissinger plots for the two steps of the crystallization processes of $\mathrm{Mg}_{70} \mathrm{Zn}_{30}$ - The slope gives the corresponding activation energies, and the values obtained are $E_{1}=153 \mathrm{~kJ} / \mathrm{mole}$, and $E_{2}=92 \mathrm{~kJ} / \mathrm{mole}$

elastic parameters of the media can be deduced:

$H=\frac{\beta E}{\rho C_{\mathrm{p}}} F(\tau, D)$,

where $\beta$ is the volume thermal expansion coefficient, $C_{\mathrm{p}}$ is the heat capacity at constant pressure, $E$ is the absorbed laser pulse energy, $\rho$ is the density, $F$ is a function of the temporal and spatial shape of the laser pulse and the geometry of the detection; $\tau$ is the laser pulse duration, and $D=2 c_{\mathrm{s}} \tau Z / a^{2}$ is the acoustic diffraction parameter, with $c_{\mathrm{s}}$ the sound velocity; $Z$ the distance between the acoustic source and the detector, and $a$ the diameter of the illuminated region.

From (1), changes in the acoustic signal can be expected from changes in the sound velocity, the alloy density, the expansion coefficient or the heat capacity. The small change measured in the sound velocity, together with the smooth dependence of $F$ with $D$, cannot explain the large variations observed in the acoustic signal. The change in the density between the glass, crystal and liquid is also very small $[14,15]$. 


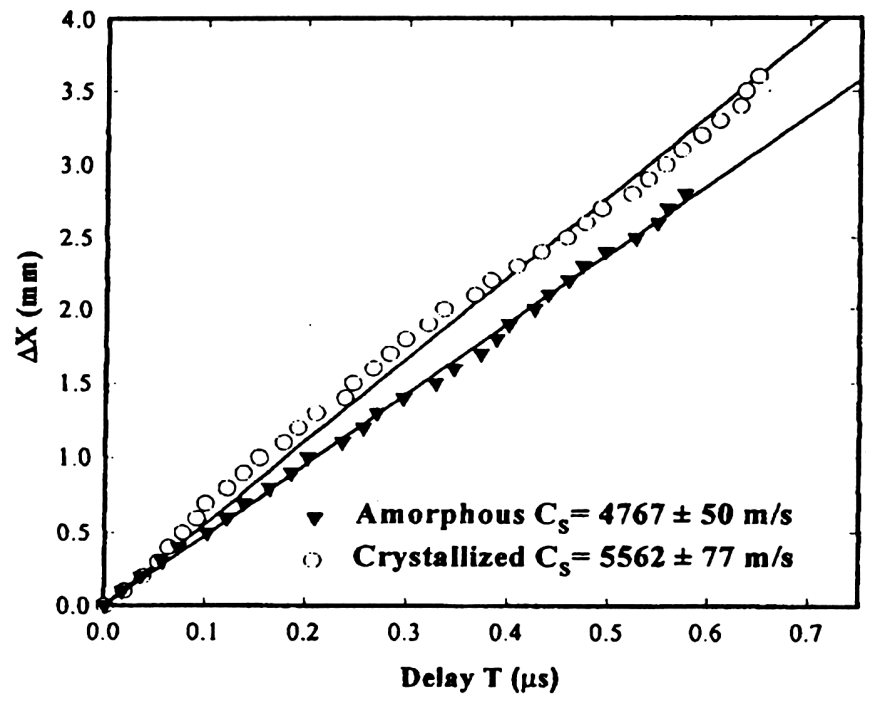

Fig. 5. Sound velocity determination in the amorphous and crystalline phase. The delay in the arrival of the acoustic signal was determined as a function of the distance between the laser spot and the position of the transducer

During a glass to undercooled liquid transition, $C_{\mathrm{p}}$ and $\beta$ are expected to increase upon heating, and conversely $C_{\mathrm{p}}$ and $\beta$ should decrease in the undercooled liquid to crystal transition $[1,2]$. Hence from (1), the sign of the change in the photoacoustic signal will depend on which of the two magnitudes has a larger relative change.

It is convenient at this stage to summarize prior results obtained by other authors with this alloy. The crystallization upon heating has been described by several authors $[10,11$, $13,18,19]$, showing first a crystallization transition around $360 \mathrm{~K}$, reflected by a decrease of the order of $10 \%$ in the electrical resistivity $[11,13,18]$, and two peaks in DSC experiments $[10,19]$. Smaller crystallization temperatures were found by Schaal [23] using small angle X-ray scattering and very slow heating rates $(0.375 \mathrm{~K} / \mathrm{min})$. This first doublet is associated with the polymorphous crystallization of very fine grained $\mathrm{Mg}_{51} \mathrm{Zn}_{20}$ which is highly defective, and a subsequent recrystallization process to more perfect grains. A much smaller DSC peak occurs at higher temperature (around $500 \mathrm{~K}$ ) due to the precipitation of some $\mathrm{Mg}$.

From the photoacoustic results, the first transition can be assigned to the material crystallization to $\mathrm{Mg}_{51} \mathrm{Zn}_{20}$ (detected as a doublet by DSC $[11,13,18])$. Our results with the electrical resistivity are similar to those previously published [10, 19], validating our interpretation of the photoacoustic signal changes. From Eq. (1), the increase of the photoacoustic sig$\mathrm{nal}$ upon crystallization indicates that the change in $C_{\mathrm{p}}$ is the dominant mechanism involved in this transition. This is consistent with the results reported in [15], where no important change in the thermal expansions in this temperature range were found during their diffraction studies.

From prior work, the activation energies of the two peaks of the DSC doublet were found to be $160 \mathrm{~kJ} / \mathrm{mole}$ and $120 \mathrm{~kJ} / \mathrm{mole}$ [11]. Shiotani et al. [18] determined the activation energy for different compositions between 69 at.\% and 72 at\% of $\mathrm{Mg}$ by Arrhenius plots using the electrical resistivity changes, yielding values between $150 \mathrm{~kJ} / \mathrm{mole}$ and $130 \mathrm{~kJ} / \mathrm{mole}$. Calka [16] showed that the activation energy depends on the composition and aging of the sample. With our technique only one activation energy $E_{1}=153 \mathrm{~kJ} / \mathrm{mole}$ was determined with a value consistent with prior works.

The second transition detected corresponds to the segregation of $\mathrm{Mg}$, as reported by several authors $[10,11,13,18,19]$. The activation energy for this transition has not been previously reported.

It was not possible to determine the glass transition from our measurement, probably due to its proximity to room temperature. The small decrease in the photoacoustic signal between room temperature and $350 \mathrm{~K}$ can be attributed to the increase in $C_{\mathrm{p}}$ during the glass transition, but this result was not repetitive enough for an unambiguous assignment. This coincides with previous findings, that showed that when scanned with DSC the endothermic glass transition is either absent or extremely weak [10].

The photoacoustic signal increases by a factor of two in the first transition while the electrical resistivity decreases by only $15 \%$, showing a larger sensitivity of the new method for this amorphous to crystalline transition. This feature arises from the large change in $C_{\mathrm{p}}$ for these transitions. For the crystal-crystal transition, the sensitivities are similar, showing a $50 \%$ relative change in both magnitudes.

\section{Conclusions}

IIt has been shown that the changes produced in the photoacoustic signal generated by low energy laser pulses (less than $200 \mu \mathrm{J})$, is a sensitive probe for detecting the crystallization of a metallic amorphous sample.

The developed technique has the following advantages over other competitive methods. A minimum amount of sample is required, and it can be solid or a powder. Due to the focusability of the laser and the negligible penetration depth of the light, small area, thin amorphous layers can be studied. No special sample preparation or conditioning is necessary. No contacts are soldered or glued and no special shape is required.

In the particular case of the alloy studied, the sensitivity of the photoacoustic measurements was higher than that obtained with electrical resistivity measurements.

Inspite of the high sensitivity of the technique, the $\mathrm{Nd}^{3+}$ YAG pulsed laser used can be replaced by smaller compact lasers such as diode pumped systems. In this way, the developed method can be converted in a very simple, compact system, to be used for in situ studies and incorporated into larger equipment such as electron microscopes, X-ray diffractometers, or scanning calorimeters.

Acknowledgements. The authors wish to thank Dr. M.E. de la Cruz for providing the melt-spinning equipment for the production of the samples, and Eng. N. Martinez for the resistivity measurement equipment. This work was carried out with partial support from the Universidad de Buenos Aires and from the Universidad Nacional de La Plata.

\section{References}

1. G.W. Scherer: Glass formation and relaxation in Mater. Sci. Technol. Vol.9, ed. by R.W. Cahn, P. Haasen, E.J. Kramer (VCH, New York 1991)

2. H. Baxi, T.B. Massalski: Mater. Sci. Eng. 97, 291 (1988)

3. H.E. Kissinger. J. Res. Nat. Bur. Stand. 57, 217 (1956) 
4. M.A. Siqueira, C.C. Ghizoni, J.I. Vargas , E.A. Menezes, H. Vargas, L.C.M. Miranda: J. Appl. Phys. 51, 1403 (1980)

5. H. Vargas, L.C.M. Miranda: Phys. Rep. (Review Section of Physics Letters) 161, 43 (1988)

6. J. Fernandez, J. Etxebarria, M.J. Tello, A. Lopez Acharri, J. Phys. D: Appl. Phys. 16, 269 (1983)

7. C. Pichon, M. Le Liboux, D. Foumier, A.C. Boccara: Appl. Phys. Lett. 35,435 (1979)

8. See for example: 8th Intenjational Topical Meeting on Photoacoustic and Photothermal Phenomena, Journal de Physique IV, Colloque C7, Vol.4 C7-545-667. (1994)

9. I. Higashi, N. Shiotani, M. Uda, T. Mizoguchi, H. Katoh: J. Solid State Chem. 36, 225 (1981)

10. A. Calka, M. Madhava, D.E. Polk, B.C. Giessen, H. Matyja, J. Vander Sande: Scripta Metall. 11, 65 (1977)

11. P. Boswell: Mater. Sci. Eng. 34, 1 (1978)

12. H. Rudin, S. Jost, H.J. Güntherodt: J. Non-Cryst. Solids 61/62, 291 (1984)

13. M. Ito, H. Narumi, T. Mizoguchi, T. Kawamura, H. Iwasaki, N. Shio- tani: J. Phys. Soc. Jpn. 54, 1843 (1985)

14. P. Andonov, P. Chieux: J. Non-Cryst. Solids 93, 331 (1987)

15. P. Andonov, P. Chieux: J. Non-Cryst. Solids 108, 58 (1989)

16. A. Calka: J. Phys. F: Met. Phys. 16, 1577 (1986)

17. J.B. Suck, H. Rudin, H.S. Güntherodt, H. Beck: J. Phys. C: Solid State Phys. 14, 2305 (1981)

18. N. Shiotani, H. Narumi, H. Arai, K. Wakatsuki, Y. Sasa, T. Mizoguchi: Proc. 4th. Int. Conf. on Rap. Quenched Metals, Sendai, Japan, 2, 667 (1981)

19. T. Matsuda, U. Mizutani: Proc. 4th. Int. Conf. on Rap. Quenched Metals, Sendai, Japan 2, 1315 (1981)

20. C.K.N. Patel, A.C. Tam: Rev. Mod. Phys. 53, 517 (1981)

21. R.W. Cahn: Metallic Glasses in Mater. Sci. Technol. Vol. 9, ed. by R.W. Cahn, P. Haasen, E.J. Kramer (VCH, New York 1991)

22. F.V. Bunkin, Al.A. Kolomensky, V.G. Mikhalevich: In Lasers in Acoustics, Chap. 4, (Harwood Academic Publishers, Switzerland 1991)

23. M. Schaal, P. Lamparter, S. Steeb: Z. Naturforsch. 41a, 1123 (1986) 